\title{
A Necessary and Sufficient Condition for the Existence of Positive Solutions to the Singular $p$-Laplacian
}

\author{
R. P. Agarwal, Haishen Lü and D. O'Regan
}

Abstract. This paper studies the boundary value problem

$$
\left.\begin{array}{rl}
\left(\varphi_{p}\left(u^{\prime}\right)\right)^{\prime}+q(t)(f(u)+g(u)) & =0 \quad(0<t<1) \\
u(0)=u(1) & =0
\end{array}\right\}
$$

in the case $p>1$. A necessary and sufficient condition for the existence of $C^{1}[0,1]$ positive solutions and a sufficient condition for the existence of $C[0,1]$ positive solutions are presented.

Keywords: Singular boundary value problems, positive solutions, existence conditions for solutions

AMS subject classification: 34B16, 39A10

\section{Introduction}

The boundary value problem

$$
\left.\begin{array}{c}
\left(\varphi_{p}\left(u^{\prime}\right)\right)^{\prime}+f(t, u)=0 \quad(0<t<1) \\
u(0)=u(1)=0
\end{array}\right\}
$$

where $\varphi_{p}(s)=|s|^{p-2} s \quad(p>1)$ has been studied extensively in the literature (see $[3-5,7-10,12,17]$ and the references therein). In this paper,

R. P. Agarwal: Florida Inst. Techn., Dept. Math. Sci., Melbourne, Florida 32901, USA; agarwal@fit.edu

Haishen Lü: Chinese Acad. Sci., Acad. Math. \& System Sci., Inst. Appl. Math., Beijing 100080, P.R. China; haishen2001@yahoo.com.cn

D. O'Regan: Nat. Univ. of Ireland, Dept. Math., Galway, Ireland

donal.oregan@ucg.ie

The research is supported by NNSF of China (19971037)

ISSN 0232-2064 / \$2.50 C Heldermann Verlag Berlin 
we establish a necessary and sufficient condition for the existence of $C^{1}[0,1]$ positive solutions and a sufficient condition for the existence of $C[0,1]$ positive solutions to the two-point boundary value problem

$$
\left.\begin{array}{rl}
\left(\varphi_{p}\left(u^{\prime}\right)\right)^{\prime}+q(t)(f(u)+g(u)) & =0 \quad(0<t<1) \\
u(0)=u(1) & =0
\end{array}\right\}
$$

with $\varphi_{p}(s)=|s|^{p-2} s, p>1, q \in C((0,1),[0, \infty))$ and $f, g \in C([0, \infty),[0, \infty))$.

We next state a fixed point theorem due to Krasnosel'skii (see, e.g., [2]) which will be needed in Sections 2 and 3 .

Theorem 1.1. Let $X$ be a Banach space, and let $K(\subset X)$ be a cone. Assume $\Omega_{1}, \Omega_{2}$ are open subsets of $X$ with $0 \in \Omega_{1}$ and $\bar{\Omega}_{1} \subset \Omega_{2}$, and let

$$
T: K \cap\left(\bar{\Omega}_{2} \backslash \Omega_{1}\right) \rightarrow K
$$

be a continuous, compact operator such that, either

(a) $\|T u\| \leq\|u\| \quad\left(u \in K \cap \partial \Omega_{1}\right)$ and $\|T u\| \geq\|u\| \quad\left(u \in K \cap \partial \Omega_{2}\right)$

or

(b) $\|T u\| \geq\|u\| \quad\left(u \in K \cap \partial \Omega_{1}\right)$ and $\|T u\| \leq\|u\| \quad\left(u \in K \cap \partial \Omega_{2}\right)$.

Then $T$ has a fixed point in $K \cap\left(\bar{\Omega}_{2} \backslash \Omega_{1}\right)$.

In this paper $X=(C[0,1],\|\cdot\|)$ with usual maximum norm will be our Banach space and

$$
K=\left\{\begin{array}{l|l}
u \in C[0,1] & \begin{array}{l}
u \text { non-negative concave and, for some } M_{u}>0, \\
u(t) \leq M_{u} t(1-t) \text { for all } t \in[0,1]
\end{array}
\end{array}\right\}
$$

will be the cone. Also, for $R>0$ we set

$$
K_{R}=\{y \in K:\|y\|<R\}
$$

We first state two known lemmas which will be needed in the following.

Lemma 1.1 [17]. Assume that $0 \leq \rho \in L^{1}(0,1), \rho \not \equiv 0$ in $(0,1)$. Suppose

$$
u \in C_{0}^{1}[0,1]=\left\{v: v, v^{\prime} \in X, v(0)=v(1)=0\right\}
$$

is the unique positive solution of the problem

$$
\left.\begin{array}{l}
-\left(\varphi_{p}\left(u^{\prime}\right)\right)^{\prime}=\rho(t) \quad(0<t<1) \\
u(0)=u(1)=0
\end{array}\right\}
$$


Then there exist constants $k \geq l>0$ such that

$$
l p(t) \leq u(t) \leq k p(t) \quad(0 \leq t \leq 1)
$$

where $p(t)=\min \{t, 1-t\}$.

Lemma $1.2[1]$. Let $u \in K$. Then $u(t) \geq t(1-t)\|u\|$ for $t \in[0,1]$.

In what follows we shall assume that

(H1) $q(t)>0$ for $t \in(0,1)$ and

$$
\int_{0}^{\frac{1}{2}} \varphi_{p}^{-1}\left(\int_{s}^{\frac{1}{2}} q(r) d r\right) d s+\int_{\frac{1}{2}}^{1} \varphi_{p}^{-1}\left(\int_{\frac{1}{2}}^{s} q(r) d r\right) d s<\infty .
$$

\section{A necessary and sufficient condition for the existence of positive solutions}

In this section we write

$$
\begin{aligned}
& f_{0}=\lim _{x \rightarrow 0^{+}} \frac{f(x)}{x^{p-1}} \\
& f_{\infty}=\lim _{x \rightarrow \infty} \frac{f(x)}{x^{p-1}} \\
& g_{0}=\lim _{x \rightarrow 0^{+}} \frac{g(x)}{x^{p-1}} \\
& g_{\infty}=\lim _{x \rightarrow \infty} \frac{g(x)}{x^{p-1}} .
\end{aligned}
$$

We begin with a result which requires either

(i) $f$ and $g$ sublinear at zero and superlinear at infinity

or

(ii) $f$ and $g$ superlinear at zero and sublinear at infinity.

Theorem 2.1. Suppose condition (H1) holds. In addition, assume the following:

(H2) $f, g$ are non-decreasing and there exist constants $\lambda, \mu>1$ such that $f(c x) \leq c^{\lambda(p-1)} f(x)$ and $g(c x) \leq c^{\mu(p-1)} g(x)$ for all $c \geq 1$.

(H3) One of the following conditions hold:

(h1) $f_{0}=0, f_{\infty}=\infty, g_{0}=0, g_{\infty}=\infty$

(h2) $f_{0}=\infty, f_{\infty}=0, g_{0}=\infty, g_{\infty}=0$.

Then a necessary and sufficient condition for problem (1.2) to have a $C^{1}[0,1]$ positive solution is that

$$
0<\int_{0}^{1} q(s)(f(e(s)+g(e(s)))) d s<\infty
$$


where $e(s)=s(1-s)$.

Proof. Necessity. Assume that $u$ is a $C^{1}[0,1]$ positive solution of problem (1.2). Then $u^{\prime}(0)$ and $u^{\prime}(1)$ are finite with $\left(\varphi_{p}\left(u^{\prime}\right)\right)^{\prime} \leq 0$ and $u(t)>0$ for $t \in(0,1)$. This implies that $\varphi_{p}\left(u^{\prime}\right)$ is non-increasing, so $u^{\prime}$ is non-increasing. Thus $u$ is a concave function. This implies that there is a constant $m>0$ such that

$$
u(t) \geq m e(t) \quad(0 \leq t \leq 1) .
$$

Let $c=\min \{1, m\}$. Now condition $(H 2)$ implies that

$$
\begin{aligned}
\int_{0}^{1} q(s)(f(e(s))+g(e(s))) d s \\
\quad \leq \int_{0}^{1} q(s)\left(f\left(c^{-1} u(s)\right)+g\left(c^{-1} u(s)\right)\right) d s \\
\quad \leq \bar{c} \int_{0}^{1} q(s)(f(u(s))+g(u(s))) d s \\
\quad=-\bar{c} \int_{0}^{1}\left(\varphi_{p}\left(u^{\prime}(s)\right)\right)^{\prime} d s \\
\quad=\bar{c}\left(\varphi_{p}\left(u^{\prime}(0)\right)-\varphi_{p}\left(u^{\prime}(1)\right)\right) \\
<\infty
\end{aligned}
$$

where $\bar{c}=\max \left\{c^{-(p-1) \lambda}, c^{-(p-1) \mu}\right\}$.

On the other hand, assume that $u$ is a $C^{1}[0,1]$ positive solution of problem (1.2). Then $q(t)(f(u)+g(u)) \neq 0$ for $(t, u) \in(0,1) \times(0, \infty)$, since otherwise problem (1.2) has only the zero solution. Without loss of generality, suppose

$$
q\left(t_{0}\right)\left(f\left(u\left(t_{0}\right)\right)+g\left(u\left(t_{0}\right)\right)\right)>0
$$

for some $t_{0} \in(0,1)$. By [16: Lemma 2], there exist a constant $M>0$ such that $u(s) \leq M e(s)$ for $s \in[0,1]$. Let $M_{1}=\max \{1, M\}$. Then $u\left(t_{0}\right) \leq M e\left(t_{0}\right) \leq$ $M_{1} e\left(t_{0}\right)$. Consequently,

$$
\begin{aligned}
0 & <q\left(t_{0}\right)\left(f\left(u\left(t_{0}\right)\right)+g\left(u\left(t_{0}\right)\right)\right) \\
& \leq q\left(t_{0}\right)\left(f\left(M_{1} e\left(t_{0}\right)\right)+g\left(M_{1} e\left(t_{0}\right)\right)\right) \\
& \leq q\left(t_{0}\right)\left(M_{1}^{\lambda(p-1)} f\left(e\left(t_{0}\right)\right)+M_{1}^{\mu(p-1)} g\left(e\left(t_{0}\right)\right)\right) \\
& \leq \max \left\{M_{1}^{\lambda(p-1)}, M_{1}^{\mu(p-1)}\right\} q\left(t_{0}\right)\left(f\left(e\left(t_{0}\right)\right)+g\left(e\left(t_{0}\right)\right)\right) .
\end{aligned}
$$

As a result, $0<q\left(t_{0}\right)\left(f\left(e\left(t_{0}\right)\right)+g\left(e\left(t_{0}\right)\right)\right)$. Now, since $f, g, q$ are continuous, there exists an interval $\left[a_{1}, b_{1}\right] \subset(0,1)$ with

$$
\int_{a_{1}}^{b_{1}} q(s)(f(e(s))+g(e(s))) d s>0 .
$$


Thus (2.1) holds.

Sufficiency. We will consider two cases.

Case 1: Suppose conditions (H1), (H2), (h1) and (2.1) hold. For all $u \in K$ and $t \in(0,1)$ define

$$
\begin{aligned}
x(t)= & \int_{0}^{t} \varphi_{p}^{-1}\left(\int_{s}^{t} q(r)(f(u(r))+g(u(r))) d r\right) d s \\
& -\int_{t}^{1} \varphi_{p}^{-1}\left(\int_{t}^{s} q(r)(f(u(r))+g(u(r))) d r\right) d s .
\end{aligned}
$$

Clearly, $x$ is continuous and non-decreasing in $(0,1)$ and $x(0+)<0<x(1-)$. Thus, $x$ has zeros in $(0,1)$.

Let $\xi$ be such a zero of $x$ in $(0,1)$. Then

$$
\begin{aligned}
\int_{0}^{\xi} & \varphi_{p}^{-1}\left(\int_{s}^{\xi} q(r)(f(u(r))+g(u(r))) d r\right) d s \\
& =\int_{\xi}^{1} \varphi_{p}^{-1}\left(\int_{\xi}^{s} q(r)(f(u(r))+g(u(r))) d r\right) d s
\end{aligned}
$$

Define the operator

$$
T: K \rightarrow C[0,1]
$$

by

$$
\begin{aligned}
& (T u)(t)= \\
& \begin{cases}\int_{0}^{t} \varphi_{p}^{-1}\left(\int_{s}^{\xi} q(r)(f(u(r))+g(u(r))) d r\right) d s & \text { if } 0 \leq t \leq \xi \\
\int_{t}^{1} \varphi_{p}^{-1}\left(\int_{\xi}^{s} q(r)(f(u(r))+g(u(r))) d r\right) d s & \text { if } \xi \leq t \leq 1\end{cases}
\end{aligned}
$$

We first prove that, for all $u \in K, y=T u \in K$ and $y(\xi)$ is the maximum value of $y$ on $[0,1]$ where $\xi$ is the above fixed zero of $x$ in $(0,1)$. Fix $u \in K$ and let the constant $M_{u}$ be such that $u(t) \leq M_{u} t(1-t)$ on $[0,1]$. From the definition of $T$,

$$
y^{\prime}(t)= \begin{cases}\varphi_{p}^{-1}\left(\int_{s}^{\xi} q(r)(f(u(r))+g(u(r))) d r\right) d s \geq 0 & \text { if } 0<t \leq \xi \\ -\varphi_{p}^{-1}\left(\int_{\xi}^{s} q(r)(f(u(r))+g(u(r))) d r\right) d s \leq 0 & \text { if } \xi \leq t<1\end{cases}
$$

is continuous and non-increasing in $(0,1)$ and $y^{\prime}(\xi)=0$. Thus $T u$ is a concave function. Moreover,

$$
q(t)(f(u(t))+g(u(t))) \leq q(r)\left(f\left(M_{u} e(t)\right)+g\left(M_{u} e(t)\right)\right)
$$


for $t \in(0,1)$. Set

$$
H_{u}(t)= \begin{cases}\int_{0}^{t} \varphi_{p}^{-1}\left(\int_{s}^{\zeta} q(r)\left(f\left(M_{u} e(r)\right)+g\left(M_{u} e(r)\right)\right) d r\right) d s & \text { if } 0 \leq t \leq \zeta \\ \int_{t}^{1} \varphi_{p}^{-1}\left(\int_{\zeta}^{s} q(r)\left(f\left(M_{u} e(r)\right)+g\left(M_{u} e(r)\right)\right) d r\right) d s & \text { if } \zeta \leq t \leq 1\end{cases}
$$

where $\zeta$ is a zero of the function

$$
\begin{aligned}
x(t)= & \int_{0}^{t} \varphi_{p}^{-1}\left(\int_{s}^{t} q(r)\left(f\left(M_{u} e(r)\right)+g\left(M_{u} e(r)\right)\right) d r\right) d s \\
& -\int_{t}^{1} \varphi_{p}^{-1}\left(\int_{t}^{s} q(r)\left(f\left(M_{u} e(r)\right)+\left(M_{u} e(r)\right)\right) d r\right) d s
\end{aligned}
$$

defined for $t \in(0,1)$. Thus

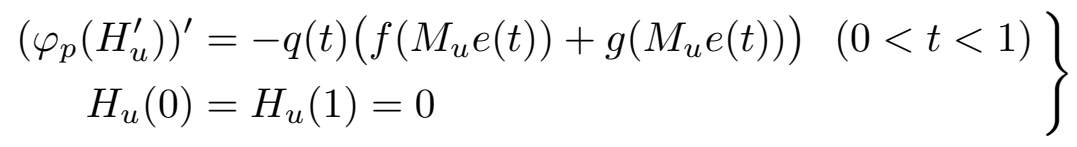

and

$$
\left.\begin{array}{rl}
\left(\varphi_{p}\left(y^{\prime}\right)\right)^{\prime} & =-q(t)(f(u(t))+g(u(t))) \quad(0<t<1) \\
y(0) & =y(1)=0
\end{array}\right\} .
$$

We claim that $y(t) \leq H_{u}(t)$ for $t \in(0,1)$. If not, there would exist $0<t_{0}<1$ with $y\left(t_{0}\right)>H_{u}\left(t_{0}\right)$, and so there would exist an interval $(a, b)$ such that $y(t)>H_{u}(t)$ for $t \in(a, b)$ and

$$
y(a)-H_{u}(a)=y(b)-H_{u}(b)=0 .
$$

Let $m=y(B)-H_{u}(B)$ be the positive maximum of $y(t)-H_{u}(t)$ on $[a, b]$. Then $B \in(a, b)$ and

$$
y^{\prime}(B)=H_{u}^{\prime}(B) .
$$

Integrating both sides of equalities (2.7) and (2.8) over $[s, B] \quad(a<s<B)$ yields

$$
\begin{aligned}
H_{u}^{\prime}(s) & =\varphi_{p}^{-1}\left(\varphi_{p}\left(H_{u}^{\prime}(B)\right)+\int_{s}^{B} q(r)\left(f\left(M_{u} e(r)\right)+g\left(M_{u} e(r)\right)\right) d r\right) \\
y^{\prime}(s) & =\varphi_{p}^{-1}\left(\varphi_{p}\left(y^{\prime}(B)\right)+\int_{s}^{B} q(r)(f(u(r))+g(u(r))) d r\right) .
\end{aligned}
$$

Integrating both equalities on $[a, B]$ yields

$$
\begin{aligned}
H_{u}(B)-H_{u}(a)= & \int_{a}^{B} \varphi_{p}^{-1}\left(\varphi_{p}\left(H_{u}^{\prime}(B)\right)\right. \\
& \left.+\int_{s}^{B} q(r)\left(f\left(M_{u} e(r)\right)+g\left(M_{u} e(r)\right)\right) d r\right) d s \\
y(B)-y(a)= & \int_{a}^{B} \varphi_{p}^{-1}\left(\varphi_{p}\left(y^{\prime}(B)\right)+\int_{s}^{B} q(r)(f(u(r))+g(u(r))) d r\right) d s .
\end{aligned}
$$


Using inequality (2.6) we have

$$
\int_{s}^{B} q(r)(f(u(r))+g(u(r))) d r \leq \int_{s}^{B} q(r)\left(f\left(M_{u} e(r)\right)+g\left(M_{u} e(r)\right)\right) d r
$$

for $s \in(a, B)$. Also, (2.10) implies $\varphi_{p}\left(H_{u}^{\prime}(B)\right)=\varphi_{p}\left(y^{\prime}(B)\right)$. Thus, for $s \in$ $(a, B)$ we have

$$
\begin{aligned}
& \varphi_{p}^{-1}\left(\varphi_{p}\left(y^{\prime}(B)\right)+\int_{s}^{B} q(r)(f(u(r))+g(u(r))) d r\right) \\
& \quad \leq \varphi_{p}^{-1}\left(\varphi_{p}\left(H_{u}^{\prime}(B)\right)+\int_{s}^{B} q(r)\left(f\left(M_{u} e(r)\right)+g\left(M_{u} e(r)\right)\right) d r\right) .
\end{aligned}
$$

Consequently, $y(B)-y(a) \leq H_{u}(B)-H_{u}(a)$. This together with (2.9) yields $y(B)-H_{u}(B) \leq 0$. We got a contraction since $y(B)-H_{u}(B)=m>0$. Thus $y(t) \leq H_{u}(t)$ for $t \in[0,1]$.

Note that, because (2.1) holds,

$$
\rho(r)=q(r)\left(f\left(M_{u} e(r)\right)+g\left(M_{u} e(r)\right)\right)
$$

satisfies the conditions of Lemma 1.1. Thus there exists a constant $k>0$ such that $y(t) \leq H_{u}(t) \leq k p(t)$ for $t \in[0,1]$. Consequently, there exist a constant $M_{y}>0$ such that $y(t) \leq M_{y} e(t)$ on $[0,1]$. This shows $T(K) \subset K$. Of course, each fixed point of $T$ in $K$ is a solution of problem (1.2).

We now claim that, for all $R>0$, the operator $T: \bar{K}_{R} \rightarrow K$ is continuous and compact. We first show that $T \bar{K}_{R}$ is bounded. For this put

$$
\begin{aligned}
Y(t)= & \varphi_{p}^{-1}(f(R)+g(R)) \\
& \times \begin{cases}\int_{0}^{t} \varphi_{p}^{-1}\left(\int_{s}^{\tau} q(r) d r\right) d s & \text { if } 0 \leq t \leq \tau \\
\int_{t}^{1} \varphi_{p}^{-1}\left(\int_{\tau}^{s} q(r) d r\right) d s & \text { if } \tau \leq t \leq 1\end{cases}
\end{aligned}
$$

where $\tau$ is a zero of the function

$$
x(t)=\int_{0}^{t} \varphi_{p}^{-1}\left(\int_{s}^{t} q(r) d r\right) d s-\int_{t}^{1} \varphi_{p}^{-1}\left(\int_{t}^{s} q(r) d r\right) d s
$$

defined for $t \in(0,1)$. Let $u \in \bar{K}_{R}$. It is clear that

$$
\begin{aligned}
\left(\varphi_{p}\left(Y^{\prime}\right)\right)^{\prime}+(f(R)+g(R)) q(t) & =0 \\
\left(\varphi_{p}\left((T u)^{\prime}\right)\right)^{\prime}+q(t)(f(u(t))+g(u(t))) & =0 \\
q(t)(f(u(t))+g(u(t))) & \leq(f(R)+g(R)) q(t)
\end{aligned}
$$


for $t \in[0,1]$. Essentially the same reasoning as above yields $0 \leq T u(t) \leq Y(t)$ for $u \in \bar{K}_{R}$ and $t \in[0,1]$. Thus the image $T \bar{K}_{R}$ is bounded.

We next show the equicontinuity of the image $T \bar{K}_{R}$ on $[0,1]$. Indeed, for any $\varepsilon>0$, from the continuity of $Y$ on $[0,1]$ it follows that there is a $\delta_{1} \in\left(0, \frac{1}{4}\right)$ such that

$$
0 \leq T u(t) \leq Y(t)<\varepsilon
$$

for every $u \in \bar{K}_{R}$ and $t \in\left[0,2 \delta_{1}\right] \cup\left[1-2 \delta_{1}, 1\right]$. Thus $\left|T u\left(t_{1}\right)-T u\left(t_{2}\right)\right|<2 \varepsilon$ for $t_{1}, t_{2} \in\left[0,2 \delta_{1}\right)$ or $t_{1}, t_{2} \in\left(1-2 \delta_{1}, 1\right]$. Next, we consider $t_{1}, t_{2} \in\left[2 \delta_{1}, 1-2 \delta_{1}\right]$. If $0 \leq T u(\xi)<\varepsilon$, then

$$
\left|T u\left(t_{1}\right)-T u\left(t_{2}\right)\right| \leq\left|T u\left(t_{1}\right)\right|+\left|T u\left(t_{2}\right)\right|<2 \varepsilon
$$

for any $t_{1}, t_{2} \in[0,1]$. If $T u(\xi) \geq \varepsilon$, then $\xi \in\left[2 \delta_{1}, 1-2 \delta_{1}\right]$ and hence for $t \in\left[\delta_{1}, 1-\delta_{1}\right]$ we have

$$
\left|(T u)^{\prime}(t)\right| \leq\left|\varphi_{p}^{-1}\left(\int_{\delta_{1}}^{1-\delta_{1}}(f(R)+g(R)) q(r) d r\right)\right|=L .
$$

Put $\delta_{2}=\frac{\varepsilon}{L}$. Then for $t_{1}, t_{2} \in\left[\delta_{1}, 1-\delta_{1}\right]$ and $\left|t_{1}-t_{2}\right|<\delta_{2}$ we have

$$
\left|T u\left(t_{1}\right)-T u\left(t_{2}\right)\right| \leq\left|(T u)^{\prime}(\eta)\right|\left|t_{1}-t_{2}\right|<L \delta_{2}=\varepsilon
$$

where $\eta$ lies between $t_{1}$ and $t_{2}$. Let $\delta=\min \left\{\delta_{1}, \delta_{2}\right\}$. Then for $t_{1}, t_{2} \in[0,1]$ with $\left|t_{1}-t_{2}\right|<\delta$ we have

$$
\left|T u\left(t_{1}\right)-T u\left(t_{2}\right)\right|<3 \varepsilon \quad\left(u \in \bar{K}_{R}\right) .
$$

This shows that $T \bar{K}_{R}$ is equicontinuous on $[0,1]$.

We next claim that $T: \bar{K}_{R} \rightarrow K$ is continuous. Assume that $\left\{u_{n}\right\}_{n=0}^{\infty} \subset$ $\bar{K}_{R}$ and $u_{n} \rightarrow u_{0}$ uniformly on [0,1]. The Arzela-Ascoli theorem guarantees that there exist a subsequences of $\left\{T u_{n}\right\}_{n=1}^{\infty}$ (without loss of generality assume it is the whole sequence) and a $v \in C[0,1]$ with $T u_{n} \rightarrow v$ uniformly on $[0,1]$ as $n \rightarrow \infty$. We can also assume without loss of generality that $\xi_{n}$ converges, and we suppose $\xi_{n} \rightarrow \xi_{0}$ as $n \rightarrow \infty$. We will now prove that $v(t)=\left(T u_{0}\right)(t)$ for $t \in[0,1]$.

Without loss of generality, we may choose a sequence $\left\{\xi_{n_{j}}\right\}$ such that $\left\{\xi_{n_{j}}\right\}$ is monotonically increasing (the proof is similar if it is monotonically decreasing) and $\xi_{n_{j}} \rightarrow \xi_{0}$ as $j \rightarrow \infty$. Then,

$$
\begin{array}{r}
\varphi_{p}^{-1}\left(\int_{s}^{\xi_{n_{j}}} q(r)\left(f\left(u_{n_{j}}(r)\right)+g\left(u_{n_{j}}(r)\right)\right) d r\right) \\
\leq \varphi_{p}^{-1}(f(R)+g(R)) \varphi_{p}^{-1}\left(\int_{s}^{\xi_{0}} q(r) d r\right)
\end{array}
$$


for $s \in\left[0, \xi_{n_{j}}\right]$. The Lebesgue's Dominated Convergence Theorem guarantees

$$
\begin{aligned}
v(t) & =\lim _{n \rightarrow \infty} T u_{n}(t) \\
& =\int_{0}^{t} \varphi_{p}^{-1}\left(\int_{s}^{\xi_{0}} q(r)\left(f\left(u_{0}(r)\right)+g\left(u_{0}(r)\right)\right) d r\right) d s
\end{aligned}
$$

for $t \in\left[0, \xi_{0}\right]$. Notice that, for any integers $j>J$,

$$
\varphi_{p}^{-1}(f(R)+g(R)) \int_{\xi_{n_{J}}}^{1} \varphi_{p}^{-1}\left(\int_{\xi_{n_{J}}}^{s} q(r) d r\right) d s<\infty
$$

and

$$
\begin{aligned}
\varphi_{p}^{-1} & \left(\int_{\xi_{n_{j}}}^{s} q(r)\left(f\left(u_{n_{j}}(r)\right)+g\left(u_{n_{j}}(r)\right)\right) d r\right) d s \\
& \leq \varphi_{p}^{-1}(f(R)+g(R)) \varphi_{p}^{-1}\left(\int_{\xi_{n_{J}}}^{s} q(r) d r\right) d s
\end{aligned}
$$

for $s \in\left[\xi_{n_{j}}, 1\right]$. The Lebesgue's Dominated Convergence Theorem guarantees that

$$
\begin{aligned}
v(t) & =\lim _{j \rightarrow \infty} \int_{t}^{1} \varphi_{p}^{-1}\left(\int_{\xi_{n_{j}}}^{s} q(r)\left(f\left(u_{n_{j}}(r)\right)+g\left(u_{n_{j}}(r)\right)\right) d r\right) d s \\
& =\int_{t}^{1} \varphi_{p}^{-1}\left(\int_{\xi_{0}}^{s} q(r)\left(f\left(u_{0}(r)\right)+g\left(u_{0}(r)\right)\right) d r\right) d s
\end{aligned}
$$

for $s \in\left[\xi_{0}, 1\right]$. From (2.14) and (2.15) we get

$$
\begin{aligned}
v\left(\xi_{0}\right) & =\int_{0}^{\xi_{0}} \varphi_{p}^{-1}\left(\int_{s}^{\xi_{0}} q(r)\left(f\left(u_{0}(r)\right)+g\left(u_{0}(r)\right)\right) d r\right) d s \\
& =\int_{\xi_{0}}^{1} \varphi_{p}^{-1}\left(\int_{\xi_{0}}^{s} q(r)\left(f\left(u_{0}(r)\right)+g\left(u_{0}(r)\right)\right) d r\right) d s
\end{aligned}
$$

Clearly, $v(t)=\left(T u_{0}\right)(t)$ for all $t \in[0,1]$.

The above shows that there exists a subsequence $S$ of $\mathbb{N}$ with $T u_{n} \rightarrow T u_{0}$ uniformly on $[0,1]$ as $n \rightarrow \infty$ in $S$. We now show $T u_{n} \rightarrow T u_{0}$ uniformly on $[0,1]$ as $n \rightarrow \infty$ in $N$. Suppose this is false. Then there exist $\varepsilon_{0}>0$ and a subsequence $S_{1}$ of $N$ with $\left|T u_{n}-T u_{0}\right|_{0} \geq \varepsilon_{0} \quad\left(n \in S_{1}\right)$. Since $u_{n} \rightarrow u_{0}$ uniformly on [0,1] as $n \rightarrow \infty$ in $S_{1}$, then as above there exists a subsequence $S_{2}$ of $S_{1}$ with $T u_{n} \rightarrow T u_{0}$ uniformly on $[0,1]$ as $n \rightarrow \infty$ in $S_{2}$. This is a contradiction. As a result, $T: \bar{K}_{R} \rightarrow K$ is a continuous, compact operator. 
We now show that problem (1.2) has a positive $C^{1}[0,1]$ solution. Since $f_{0}=g_{0}=0$, there exist a constant $l>0$ such that

$$
\begin{aligned}
& f(u) \leq \eta^{p-1} u^{p-1} \\
& g(u) \leq \eta^{p-1} u^{p-1}
\end{aligned} \quad(0<u \leq l)
$$

where $\eta$ satisfies

$$
2^{\frac{1}{p-1}} \eta \max \left\{\int_{0}^{\frac{1}{2}} \varphi_{p}^{-1}\left(\int_{s}^{\frac{1}{2}} q(r) d r\right) d s, \int_{\frac{1}{2}}^{1} \varphi_{p}^{-1}\left(\int_{\frac{1}{2}}^{s} q(r) d r\right) d s\right\} \leq 1 .
$$

If $u \in K$ and $\|u\|=l$, then for $0 \leq t \leq 1$ we have (with $\xi$ and $T$ defined in (2.4) and (2.5))

$$
\begin{aligned}
& (T u)(t)= \begin{cases}\int_{0}^{t} \varphi_{p}^{-1}\left(\int_{s}^{\xi} q(r)(f(u(r))+g(u(r))) d r\right) d s & \text { if } 0 \leq t \leq \xi \\
\int_{t}^{1} \varphi_{p}^{-1}\left(\int_{\xi}^{s} q(r)(f(u(r))+g(u(r))) d r\right) d s & \text { if } \xi \leq t \leq 1\end{cases} \\
& \leq \int_{0}^{\xi} \varphi_{p}^{-1}\left(\int_{s}^{\xi} q(r)(f(u(r))+g(u(r))) d r\right) d s \\
& =\int_{\xi}^{1} \varphi_{p}^{-1}\left(\int_{\xi}^{s} q(r)(f(u(r))+g(u(r))) d r\right) d s \\
& \leq \max \left\{\begin{array}{l}
\int_{0}^{\frac{1}{2}} \varphi_{p}^{-1}\left(\int_{s}^{\frac{1}{2}} q(r)(f(u(r))+g(u(r))) d r\right) d s \\
\int_{\frac{1}{2}}^{1} \varphi_{p}^{-1}\left(\int_{\frac{1}{2}}^{s} q(r)(f(u(r))+g(u(r))) d r\right) d s
\end{array}\right\} \\
& \leq \max \left\{\begin{array}{l}
\int_{0}^{\frac{1}{2}} \varphi_{p}^{-1}\left(\int_{s}^{\frac{1}{2}} q(r)\left(\eta^{p-1}(u(r))^{p-1}+\eta^{p-1}(u(r))^{p-1}\right) d r\right) d s \\
\int_{\frac{1}{2}}^{1} \varphi_{p}^{-1}\left(\int_{\frac{1}{2}}^{s} q(r)\left(\eta^{p-1}(u(r))^{p-1}+\eta^{p-1}(u(r))^{p-1}\right) d r\right) d s
\end{array}\right\} \\
& \leq \max \left\{\begin{array}{l}
\int_{0}^{\frac{1}{2}} \varphi_{p}^{-1}\left(\int_{s}^{\frac{1}{2}} 2 \eta^{p-1}\|u\|^{p-1} q(r) d r\right) d s \\
\int_{\frac{1}{2}}^{1} \varphi_{p}^{-1}\left(\int_{\frac{1}{2}}^{s} 2 \eta^{p-1}\|u\|^{p-1} q(r) d r\right) d s
\end{array}\right\} \\
& \leq 2^{\frac{1}{p-1}} \eta\|u\| \max \left\{\begin{array}{l}
\int_{0}^{\frac{1}{2}} \varphi_{p}^{-1}\left(\int_{s}^{\frac{1}{2}} q(r) d r\right) d s \\
\int_{\frac{1}{2}}^{1} \varphi_{p}^{-1}\left(\int_{\frac{1}{2}}^{s} q(r) d r\right) d s
\end{array}\right\} \\
& \leq\|u\|
\end{aligned}
$$


That is,

$$
(T u)(t) \leq\|u\| \quad(0 \leq t \leq 1) .
$$

Let $\Omega_{1}=\{u \in X:\|u\|<l\}$. Now by (2.16) we have $\|T u\| \leq\|u\|$ for all $u \in K \cap \partial \Omega_{1}$. Also, since $f_{\infty}=g_{\infty}=\infty$, there exist $L_{1}>l>0$ such that, for all $u \geq L_{1}, f(u) \geq \nu^{p-1} u^{p-1}$ and $g(u) \geq \nu^{p-1} u^{p-1}$ where $\nu$ satisfies

$$
2^{\frac{1}{p-1}} \frac{3}{16} \nu \min \left\{\int_{\frac{1}{4}}^{\frac{1}{2}} \varphi_{p}^{-1}\left(\int_{s}^{\frac{1}{2}} q(r) d r\right) d s, \int_{\frac{1}{2}}^{\frac{3}{4}} \varphi_{p}^{-1}\left(\int_{\frac{1}{2}}^{s} q(r) d r\right) d s\right\} \geq 1 .
$$

Let $L=\frac{16 L_{1}}{3}$ and $\Omega_{2}=\{u \in K:\|u\|<L\}$. If $u \in K \cap \partial \Omega_{2}$, then by Lemma 1.2 we have $\min _{\frac{1}{4} \leq s \leq \frac{3}{4}} u(s) \geq \frac{3}{16}\|u\|$ and so (with $\xi$ and $T$ defined in (2.4) and (2.5))

$$
\begin{aligned}
&\|T u\|=\int_{0}^{\xi} \varphi_{p}^{-1}\left(\int_{s}^{\xi} q(r)(f(u(r))+g(u(r))) d r\right) d s \\
&=\int_{\xi}^{1} \varphi_{p}^{-1}\left(\int_{\xi}^{s} q(r)(f(u(r))+g(u(r))) d r\right) d s \\
& \geq \min \left\{\begin{array}{l}
\left.\int_{0}^{\frac{1}{2}} \varphi_{p}^{-1}\left(\int_{s}^{\frac{1}{2}} q(r)(f(u(r))+g(u(r))) d r\right) d s\right\} \\
\int_{\frac{1}{2}}^{1} \varphi_{p}^{-1}\left(\int_{\frac{1}{2}}^{s} q(r)(f(u(r))+g(u(r))) d r\right) d s
\end{array}\right\} \\
& \geq \min \left\{\begin{array}{l}
\int_{\frac{1}{4}}^{\frac{1}{2}} \varphi_{p}^{-1}\left(\int_{s}^{\frac{1}{2}} q(r)(f(u(r))+g(u(r))) d r\right) d s \\
\int_{\frac{1}{2}}^{\frac{3}{4}} \varphi_{p}^{-1}\left(\int_{\frac{1}{2}}^{s} q(r)(f(u(r))+g(u(r))) d r\right) d s
\end{array}\right\} \\
& \geq \min \left\{\begin{array}{l}
\int_{\frac{1}{4}}^{\frac{1}{2}} \varphi_{p}^{-1}\left(\int_{s}^{\frac{1}{2}} q(r)\left(f\left(\frac{3}{16}\|u\|\right)+g\left(\frac{3}{16}\|u\|\right)\right) d r\right) d s \\
\int_{\frac{1}{2}}^{\frac{3}{4}} \varphi_{p}^{-1}\left(\int_{\frac{1}{2}}^{s} q(r)\left(f\left(\frac{3}{16}\|u\|\right)+g\left(\frac{3}{16}\|u\|\right)\right) d r\right) d s
\end{array}\right\} \\
& \geq \min \left\{\begin{array}{l}
\int_{\frac{1}{4}}^{\frac{1}{2}} \varphi_{p}^{-1}\left(\int_{s}^{\frac{1}{2}} 2 q(r) \nu^{p-1}\left(\frac{3}{16}\|u\|\right)^{p-1} d r\right) d s \\
\int_{\frac{1}{2}}^{\frac{3}{4}} \varphi_{p}^{-1}\left(\int_{\frac{1}{2}}^{s} 2 q(r) \nu^{p-1}\left(\frac{3}{16}\|u\|\right)^{p-1} d r\right) d s
\end{array}\right\}
\end{aligned}
$$




$$
\begin{aligned}
& \geq 2^{\frac{1}{p-1}} \frac{3}{16} \nu\|u\| \min \left\{\begin{array}{l}
\int_{\frac{1}{4}}^{\frac{1}{2}} \varphi_{p}^{-1}\left(\int_{s}^{\frac{1}{2}} q(r) d r\right) d s \\
\int_{\frac{1}{2}}^{\frac{3}{4}} \varphi_{p}^{-1}\left(\int_{\frac{1}{2}}^{s} q(r) d r\right) d s
\end{array}\right\} \\
& \geq\|u\| .
\end{aligned}
$$

Thus $\|T u\| \geq\|u\|$ for all $u \in K \cap \partial \Omega_{2}$. Consequently, problem (1.2) has at least one solution $u \in C[0,1] \cap C^{1}(0,1)$ with $\varphi_{p}\left(u^{\prime}\right) \in C^{1}(0,1)$ and $l \leq\|u\| \leq L$.

We next prove that $u^{\prime}(0+)$ and $u^{\prime}(1-)$ are finite. Indeed, since $u \in K$, there exists a constant $A \geq 1$ with $u(s) \leq A e(s)$ for $s \in[0,1]$. Notice that

$$
\begin{aligned}
u^{\prime}(0+) & =\lim _{t \rightarrow 0^{+}} \frac{u(t)}{t} \\
& =\lim _{t \rightarrow 0^{+}} \varphi_{p}^{-1}\left(\int_{t}^{\xi} q(r)(f(u(r))+g(u(r))) d r\right) \\
& =\varphi_{p}^{-1}\left(\int_{0}^{\xi} q(r)(f(u(r))+g(u(r))) d r\right) \\
& \leq \varphi_{p}^{-1}\left(\int_{0}^{\xi} q(r)(f(A e(r))+g(A e(r))) d r\right) \\
& \leq \bar{A} \varphi_{p}^{-1}\left(\int_{0}^{\xi} q(r)(f(e(r))+g(e(r))) d r\right) \\
& <\infty
\end{aligned}
$$

where $\bar{A}=\max \left\{A^{\lambda}, A^{\mu}\right\}$. A similar argument guarantees that $u^{\prime}(1-)$ is finite. This implies that $u$ is a $C^{1}$ positive solution of problem (1.2).

Case 2: Suppose conditions (H1), (H2) and (h2) hold. Then a slight modification of the argument in Case 1 establishes the result

In comparison to Theorem 2.1 our next result only requires one of the following conditions:

(i) $f$ is superlinear at infinity

(ii) $g$ is superlinear at infinity

(iii) $f$ is superlinear at zero

(iv) $g$ is superlinear at zero.

However, to achieve this a price has to be paid, i.e. we need to assume that $f(1)+g(1)$ is sufficiently small (see condition (H4) below).

Theorem 2.2. Suppose conditions (H1) - (H2) hold. In addition, assume the following: 
(H4) $M^{\frac{1}{p-1}} \max \left\{\int_{0}^{\frac{1}{2}} \varphi_{p}^{-1}\left(\int_{s}^{\frac{1}{2}} q(r) d r\right) d s, \int_{\frac{1}{2}}^{1} \varphi_{p}^{-1}\left(\int_{\frac{1}{2}}^{s} q(r) d r\right) d s\right\} \leq 1$ where $M=f(1)+g(1)$.

(H5) One of the following conditions hold:

(h3) $f_{\infty}=\infty$

(h4) $g_{\infty}=\infty$

(h5) $f_{0}=\infty$

(h6) $g_{0}=\infty$.

Then a necessary and sufficient condition for problem (1.2) to have a $C^{1}[0,1]$ positive solution is that

$$
0<\int_{0}^{1} q(s)(f(e(s)+g(e(s)))) d s<\infty
$$

where $e(s)=s(1-s)$.

Proof. Necessity. Essentially the same reasoning as in Theorem 2.1 establishes the result.

Sufficiency. We will consider 4 different cases.

Case (1): Suppose conditions (H1), (H2), (H4) and (h3) hold. As in Theorem 2.1 we have for all $R>0$ that $T: \bar{K}_{R} \rightarrow K$ is a continuous, compact operator. Since $f_{\infty}=\infty$, there exists $R_{1}>1$ such that $f(u) \geq \eta_{1}^{p-1} u^{p-1}$ for $u \geq R_{1}$ where $\eta_{1}>0$ and

$$
\frac{3 \eta_{1}}{16} \min \left\{\int_{\frac{1}{4}}^{\frac{1}{2}} \varphi_{p}^{-1}\left(\int_{s}^{\frac{1}{2}} q(r) d r\right) d s, \int_{\frac{1}{2}}^{\frac{3}{4}} \varphi_{p}^{-1}\left(\int_{\frac{1}{2}}^{s} q(r) d r\right) d s\right\} \geq 1
$$

Let $R_{2}=\frac{16 R_{1}}{3}$ and $\Omega_{1}=\left\{u \in X:\|u\|<R_{2}\right\}$. If $u \in K \cap \partial \Omega_{1}$, then by Lemma $1.2 \min _{\frac{1}{4} \leq s \leq \frac{3}{4}} u(s) \geq \frac{3}{16}\|u\|$ and so (with $\xi$ and $T$ defined in (2.4) and $(2.5))$

$$
\begin{aligned}
\|T u\| & =\int_{0}^{\xi} \varphi_{p}^{-1}\left(\int_{s}^{\xi} q(r)(f(u(r))+g(u(r))) d r\right) d s \\
& =\int_{\xi}^{1} \varphi_{p}^{-1}\left(\int_{\xi}^{s} q(r)(f(u(r))+g(u(r))) d r\right) d s \\
& \geq \min \left\{\begin{array}{l}
\int_{0}^{\frac{1}{2}} \varphi_{p}^{-1}\left(\int_{s}^{\frac{1}{2}} q(r)(f(u(r))+g(u(r))) d r\right) d s \\
\int_{\frac{1}{2}}^{1} \varphi_{p}^{-1}\left(\int_{\frac{1}{2}}^{s} q(r)(f(u(r))+g(u(r))) d r\right) d s
\end{array}\right\}
\end{aligned}
$$




$$
\begin{aligned}
& \geq \min \left\{\begin{array}{c}
\int_{0}^{\frac{1}{2}} \varphi_{p}^{-1}\left(\int_{s}^{\frac{1}{2}} q(r) f(u(r)) d r\right) d s \\
\int_{\frac{1}{2}}^{1} \varphi_{p}^{-1}\left(\int_{\frac{1}{2}}^{s} q(r) f(u(r)) d r\right) d s
\end{array}\right\} \\
& \geq \min \left\{\begin{array}{l}
\int_{\frac{1}{4}}^{\frac{1}{2}} \varphi_{p}^{-1}\left(\int_{s}^{\frac{1}{2}} q(r) f(u(r)) d r\right) d s \\
\int_{\frac{1}{2}}^{\frac{3}{4}} \varphi_{p}^{-1}\left(\int_{\frac{1}{2}}^{s} q(r) f(u(r)) d r\right) d s
\end{array}\right\} \\
& \geq \frac{3 \eta_{1}}{16}\|u\| \min \left\{\begin{array}{l}
\int_{\frac{1}{4}}^{\frac{1}{2}} \varphi_{p}^{-1}\left(\int_{s}^{\frac{1}{2}} q(r) d r\right) d s \\
\int_{\frac{1}{2}}^{3 / 4} \varphi_{p}^{-1}\left(\int_{\frac{1}{2}}^{s} q(r) d r\right) d s
\end{array}\right\} \\
& \geq\|u\| \text {. }
\end{aligned}
$$

Thus

$$
\|T u\| \geq\|u\| \quad\left(u \in K \cap \partial \Omega_{1}\right) .
$$

In addition, if $u \in K$ and $\|u\|=1$, then $u(s) \leq 1$ for $s \in[0,1]$. Thus we have (with $\xi$ and $T$ defined in (2.4) and (2.5))

$$
\begin{aligned}
& (T u)(t)= \begin{cases}\int_{0}^{t} \varphi_{p}^{-1}\left(\int_{s}^{\xi} q(r)(f(u(r))+g(u(r))) d r\right) d s & \text { if } 0 \leq t \leq \xi \\
\int_{t}^{1} \varphi_{p}^{-1}\left(\int_{\xi}^{s} q(r)(f(u(r))+g(u(r))) d r\right) d s & \text { if } \xi \leq t \leq 1\end{cases} \\
& \leq \int_{0}^{\xi} \varphi_{p}^{-1}\left(\int_{s}^{\xi} q(r)(f(u(r))+g(u(r))) d r\right) d s \\
& =\int_{\xi}^{1} \varphi_{p}^{-1}\left(\int_{\xi}^{s} q(r)(f(u(r))+g(u(r))) d r\right) d s \\
& \leq \max \left\{\begin{array}{l}
\int_{0}^{\frac{1}{2}} \varphi_{p}^{-1}\left(\int_{s}^{\frac{1}{2}} q(r)(f(u(r))+g(u(r))) d r\right) d s \\
\int_{\frac{1}{2}}^{1} \varphi_{p}^{-1}\left(\int_{\frac{1}{2}}^{s} q(r)(f(u(r))+g(u(r))) d r\right) d s
\end{array}\right\} \\
& \leq \max \left\{\begin{array}{c}
\int_{0}^{\frac{1}{2}} \varphi_{p}^{-1}\left(\int_{s}^{\frac{1}{2}} q(r)(f(1)+g(1)) d r\right) d s \\
\int_{\frac{1}{2}}^{1} \varphi_{p}^{-1}\left(\int_{\frac{1}{2}}^{s} q(r)(f(1)+g(1)) d r\right) d s
\end{array}\right\}
\end{aligned}
$$




$$
\begin{aligned}
& \leq M^{\frac{1}{p-1}} \max \left\{\begin{array}{l}
\int_{0}^{\frac{1}{2}} \varphi_{p}^{-1}\left(\int_{s}^{\frac{1}{2}} q(r) d r\right) d s \\
\int_{\frac{1}{2}}^{1} \varphi_{p}^{-1}\left(\int_{\frac{1}{2}}^{s} q(r) d r\right) d s
\end{array}\right\} \\
& \leq 1=\|u\| .
\end{aligned}
$$

Let $\Omega_{2}=\{u \in K:\|u\|<1\}$. Then

$$
\|T u\| \leq\|u\| \quad\left(u \in K \cap \partial \Omega_{2}\right) .
$$

By Theorem 1.1, $T$ has a fixed point $u$ in $K \cap\left(\bar{\Omega}_{1} \backslash \Omega_{2}\right)$. As in Theorem 2.1, $u$ is a positive $C^{1}[0,1]$ solution of problem (1.2).

Case (2): Suppose conditions (H1) - (H2), (H4) and (h4) hold. Then a slight modification of the argument in Case (1) establishes the result.

Case (3): Suppose conditions (H1) - H2), (H4) and (h5) hold. As in Theorem 2.1, we have that, for all $R>0, T: \bar{K}_{R} \rightarrow K$ is a continuous, compact operator. Since $f_{0}=\infty$, there exists $0<R_{1}<1$ such that $f(u) \geq \xi_{1}^{p-1} u^{p-1}$ for $0 \leq u \leq R_{1}$ where $\xi_{1}>0$ and

$$
\frac{3 \xi_{1}}{16} \min \left\{\int_{\frac{1}{4}}^{\frac{1}{2}} \varphi_{p}^{-1}\left(\int_{s}^{\frac{1}{2}} q(r) d r\right) d s, \int_{\frac{1}{2}}^{3 / 4} \varphi_{p}^{-1}\left(\int_{\frac{1}{2}}^{s} q(r) d r\right) d s\right\} \geq 1
$$

Let $\Omega_{1}=\left\{u \in X:\|u\|<R_{1}\right\}$. If $u \in K \cap \partial \Omega_{1}$, then by Lemma 1.2

$$
\min _{\frac{1}{4} \leq s \leq \frac{3}{4}} u(s) \geq \frac{3}{16}\|u\|
$$

and so we have (with $\xi$ and $T$ defined in (2.4) and (2.5))

$$
\begin{aligned}
\|T u\| & =\int_{0}^{\xi} \varphi_{p}^{-1}\left(\int_{s}^{\xi} q(r)(f(u(r))+g(u(r))) d r\right) d s \\
& =\int_{\xi}^{1} \varphi_{p}^{-1}\left(\int_{\xi}^{s} q(r)(f(u(r))+g(u(r))) d r\right) d s \\
& \geq \min \left\{\begin{array}{l}
\int_{0}^{\frac{1}{2}} \varphi_{p}^{-1}\left(\int_{s}^{\frac{1}{2}} q(r)(f(u(r))+g(u(r))) d r\right) d s \\
\int_{\frac{1}{2}}^{1} \varphi_{p}^{-1}\left(\int_{\frac{1}{2}}^{s} q(r)(f(u(r))+g(u(r))) d r\right) d s
\end{array}\right\}
\end{aligned}
$$




$$
\begin{aligned}
& \geq \min \left\{\begin{array}{l}
\left.\int_{0}^{\frac{1}{2}} \varphi_{p}^{-1}\left(\int_{s}^{\frac{1}{2}} q(r) f(u(r)) d r\right) d s\right\} \\
\left.\int_{\frac{1}{2}}^{1} \varphi_{p}^{-1}\left(\int_{\frac{1}{2}}^{s} q(r) f(u(r)) d r\right) d s\right\}
\end{array}\right. \\
& \geq \min \left\{\begin{array}{l}
\left.\int_{\frac{1}{4}}^{\frac{1}{2}} \varphi_{p}^{-1}\left(\int_{s}^{\frac{1}{2}} q(r) f(u(r)) d r\right) d s\right\} \\
\left.\int_{\frac{1}{2}}^{\frac{3}{4}} \varphi_{p}^{-1}\left(\int_{\frac{1}{2}}^{s} q(r) f(u(r)) d r\right) d s\right\} \\
\geq \min \left\{\begin{array}{l}
\int_{\frac{1}{4}}^{\frac{1}{2}} \varphi_{p}^{-1}\left(\int_{s}^{\frac{1}{2}} q(r) \xi_{1}^{p-1}(u(r))^{p-1} d r\right) d s \\
\int_{\frac{1}{2}}^{\frac{3}{4}} \varphi_{p}^{-1}\left(\int_{\frac{1}{2}}^{s} q(r) \xi_{1}^{p-1}(u(r))^{p-1} d r\right) d s
\end{array}\right\} \\
\geq \frac{3 \xi_{1}}{16}\|u\| \min \left\{\int_{\frac{1}{4}}^{\frac{1}{2}} \varphi_{p}^{-1}\left(\int_{s}^{\frac{1}{2}} q(r) d r\right) d s\right\} \\
\int_{\frac{1}{2}}^{\frac{3}{4}} \varphi_{p}^{-1}\left(\int_{\frac{1}{2}}^{s} q(r) d r\right) d s
\end{array}\right\}
\end{aligned}
$$

Thus

$$
\|T u\| \geq\|u\| \quad\left(u \in K \cap \partial \Omega_{1}\right) .
$$

In addition, as in Case (1), with $\Omega_{2}=\{u \in K:\|u\|<1\}$ we have

$$
\|T u\| \leq\|u\| \quad\left(u \in K \cap \partial \Omega_{2}\right) .
$$

By Theorem 1.1, T has a fixed point $u$ in $K \cap\left(\bar{\Omega}_{2} \backslash \Omega_{1}\right)$. As in Theorem 2.1, $u$ is a positive $C^{1}[0,1]$ solutions of problem $(1.2)$.

Case (4) : Suppose conditions (H1) - (H2), (H4) and (h6) hold. A slight modification of the argument in Case (3) establishes the result

Remark 2.1. The ideas in this section extend to the boundary value problems

$$
\left.\begin{array}{rl}
\left(\varphi_{p}\left(u^{\prime}\right)\right)^{\prime}+f(t, u) & =0 \quad(0<t<1) \\
u^{\prime}(0)=u(1) & =0
\end{array}\right\}
$$

and

$$
\left.\begin{array}{rl}
\left(\varphi_{p}\left(u^{\prime}\right)\right)^{\prime}+f(t, u) & =0 \quad(0<t<1) \\
u(0)=u^{\prime}(1) & =0
\end{array}\right\} .
$$


Minor adjustments are only needed, so we leave the details to the reader.

The conditions in Theorems 2.1 and 2.2 are easy to check in practice as the following example shows.

Example 2.1. Consider the boundary value problem

$$
\left.\begin{array}{rl}
\left(\varphi_{p}\left(u^{\prime}\right)\right)^{\prime}+q(t)\left[u^{a}+u^{b}\right] & =0 \quad(0<t<1) \\
u(0)=u(1) & =0
\end{array}\right\}
$$

with $q \in C((0,1),[0, \infty))$ and condition (H1) holding. Also, assume $a>p-1$ and $b>p-1$ and

$$
0<\int_{0}^{1} q(s)\left[s^{a}(1-s)^{a}+s^{b}(1-s)^{b}\right] d s<\infty
$$

Then problem (2.17) has a $C^{1}[0,1]$ positive solution.

We will apply Theorem 2.1 with $f(x)=x^{a}$ and $g(x)=x^{b}$. Clearly, condition (H2) holds (with $\lambda=\frac{a}{p-1}$ and $\mu=\frac{b}{p-1}$ ). Also,

$$
f_{0}=\lim _{x \rightarrow 0^{+}} x^{a-(p-1)}=0, \quad f_{\infty}=\lim _{x \rightarrow \infty} x^{a-(p-1)}=\infty, \quad g_{0}=0, \quad g_{\infty}=\infty .
$$

Thus condition (h1) holds.

\section{A sufficient condition for the existence of positive solutions}

In certain situations it is possible to replace the sublinear and superlinear conditions in the previous section by more general conditions. The main result will be presented in Theorem 3.1.

In this section, we write

$$
\begin{aligned}
& C_{1}=\max \left\{\int_{0}^{\frac{1}{2}} \varphi_{p}^{-1}\left(\int_{s}^{\frac{1}{2}} q(r) d r\right) d s, \int_{\frac{1}{2}}^{1} \varphi_{p}^{-1}\left(\int_{\frac{1}{2}}^{s} q(r) d r\right) d s\right\} \\
& C_{2}=\min \left\{\int_{\frac{1}{4}}^{\frac{1}{2}} \varphi_{p}^{-1}\left(\int_{s}^{\frac{1}{2}} q(r) d r\right) d s, \int_{\frac{1}{2}}^{\frac{3}{4}} \varphi_{p}^{-1}\left(\int_{\frac{1}{2}}^{s} q(r) d r\right) d s\right\} .
\end{aligned}
$$

Theorem 3.1. Suppose condition $\left(\mathrm{H}_{1}\right)$ holds. In addition, assume the following:

(H6) $f, g$ are non-decreasing. 
(H7) There exists a constant $r>0$ with $f(r)+g(r) \leq \varphi_{p}\left(r C_{1}\right)$.

(H8) One of the following conditions holds:

(h7) For some $R_{1}>0$ with $R_{1}>r, f\left(\frac{3 R_{1}}{16}\right)+g\left(\frac{3 R_{1}}{16}\right) \geq \varphi_{p}\left(\frac{R_{1}}{C_{2}}\right)$.

(h8) For some $R_{2}>0$ with $R_{2}<r, f\left(\frac{3 R_{2}}{16}\right)+g\left(\frac{3 R_{2}}{16}\right) \geq \varphi_{p}\left(\frac{R_{2}}{C_{2}}\right)$.

Then, problem $(1.2)$ has a positive solution $u \in C[0,1] \cap C^{1}(0,1)$ with $\phi_{p}\left(u^{\prime}\right) \in$ $C^{1}(0,1)$. In addition,

if (h7) holds, then $r \leq\|u\| \leq R_{1}$ with $u(t) \geq r t(1-t)$ for $t \in[0,1]$

if (h8) holds, then $R_{2} \leq\|u\| \leq r$ with $u(t) \geq R_{2} t(1-t)$ for $t \in[0,1]$.

Proof. Let

$$
K=\{u \in C[0,1]: u \text { non-negative and concave }\} .
$$

As in Section 2, for all $u \in K$ define

$$
\begin{aligned}
x(t)= & \int_{0}^{t} \varphi_{p}^{-1}\left(\int_{s}^{t} q(r)(f(u(r))+g(u(r))) d r\right) d s \\
& -\int_{t}^{1} \varphi_{p}^{-1}\left(\int_{t}^{s} q(r)(f(u(r))+g(u(r))) d r\right) d s
\end{aligned}
$$

for $0<t<1$. Clearly, $x$ is continuous and non-decreasing in $(0,1)$ and $x(0+)<0<x(1-)$. Thus, $x$ has zeros in $(0,1)$.

Let $\xi$ be such a zero of $x$ in $(0,1)$. Then

$$
\begin{aligned}
\int_{0}^{\xi} & \varphi_{p}^{-1}\left(\int_{s}^{\xi} q(r)(f(u(r))+g(u(r))) d r\right) d s \\
& =\int_{\xi}^{1} \varphi_{p}^{-1}\left(\int_{\xi}^{s} q(r)(f(u(r))+g(u(r))) d r\right) d s .
\end{aligned}
$$

Define the operator

$$
T: K \rightarrow C[0,1]
$$

by

$$
\begin{aligned}
& (T u)(t)= \\
& \begin{cases}\int_{0}^{t} \varphi_{p}^{-1}\left(\int_{s}^{\xi} q(r)(f(u(r))+g(u(r))) d r\right) d s & \text { if } 0 \leq t \leq \xi \\
\int_{t}^{1} \varphi_{p}^{-1}\left(\int_{\xi}^{s} q(r)(f(u(r))+g(u(r))) d r\right) d s & \text { if } \xi \leq t \leq 1 .\end{cases}
\end{aligned}
$$

Essentially the same reasoning as in Section 2 guarantees that, for all $R>0$, $T: \bar{K}_{R} \rightarrow K$ is a continuous, compact operator. Let

$$
\Omega_{1}=\{u \in X:\|u\|<r\} .
$$


We first show

$$
\|T u\| \leq\|u\| \quad\left(u \in K \cap \partial K_{r}\right)
$$

To see this let $u \in K \cap \partial K_{r}$. Then $\|u\|=r$ and $u(t) \leq r$ for $t \in[0,1]$. Then

$$
\begin{aligned}
T u(t) & =\left\{\begin{array}{l}
\int_{0}^{t} \varphi_{p}^{-1}\left(\int_{s}^{\xi} q(r)(f(u(r))+g(u(r))) d r\right) d s \quad \text { if } 0 \leq t \leq \xi \\
\int_{t}^{1} \varphi_{p}^{-1}\left(\int_{\xi}^{s} q(r)(f(u(r))+g(u(r))) d r\right) d s \quad \text { if } \xi \leq t \leq 1 .
\end{array}\right. \\
& \leq \int_{0}^{\xi} \varphi_{p}^{-1}\left(\int_{s}^{\xi} q(r)(f(u(r))+g(u(r))) d r\right) d s \\
& =\int_{\xi}^{1} \varphi_{p}^{-1}\left(\int_{\xi}^{s} q(r)(f(u(r))+g(u(r))) d r\right) d s \\
& \leq \max ^{\frac{1}{2}}\left\{\begin{array}{l}
\int_{0}^{-1}\left(\int_{s}^{\frac{1}{2}} q(r)(f(u(r))+g(u(r))) d r\right) d s \\
\int_{\frac{1}{2}}^{1} \varphi_{p}^{-1}\left(\int_{\frac{1}{2}}^{s} q(r)(f(u(r))+g(u(r))) d r\right) d s
\end{array}\right\} \\
& \leq \varphi_{p}^{-1}(f(r)+g(r)) \max \left\{\int_{0}^{\frac{1}{2}} \varphi_{p}^{-1}\left(\int_{s}^{\frac{1}{2}} q(r) d r\right) d s\right) \\
\leq & \frac{r}{C_{1}} \cdot C_{1}=r=\|u\| .
\end{aligned}
$$

This implies that $\|T u\| \leq\|u\|$.

Case (1): Assume condition (h7) holds. Let $\Omega_{2}=\left\{u \in X:\|u\|<R_{1}\right\}$. We show

$$
\|T u\| \geq\|u\| \quad\left(u \in K \cap \partial \Omega_{2}\right) .
$$

If $u \in K \cap \partial \Omega_{2}$, then $\|u\|=R_{1}$, and so from Lemma 1.2 we have $u(s) \geq \frac{3}{16} R_{1}$ for $t \in\left[\frac{1}{4}, \frac{3}{4}\right]$. Then

$$
\begin{aligned}
\|T u\| & =\int_{0}^{\xi} \varphi_{p}^{-1}\left(\int_{s}^{\xi} q(r)(f(u(r))+g(u(r))) d r\right) d s \\
& =\int_{\xi}^{1} \varphi_{p}^{-1}\left(\int_{\xi}^{s} q(r)(f(u(r))+g(u(r))) d r\right) d s
\end{aligned}
$$




$$
\begin{aligned}
& \geq \min \left\{\begin{array}{l}
\int_{0}^{\frac{1}{2}} \varphi_{p}^{-1}\left(\int_{s}^{\frac{1}{2}} q(r)(f(u(r))+g(u(r))) d r\right) d s \\
\int_{\frac{1}{2}}^{1} \varphi_{p}^{-1}\left(\int_{\frac{1}{2}}^{s} q(r)(f(u(r))+g(u(r))) d r\right) d s
\end{array}\right\} \\
& \geq \min \left\{\begin{array}{l}
\int_{\frac{1}{4}}^{\frac{1}{2}} \varphi_{p}^{-1}\left(\int_{s}^{\frac{1}{2}} q(r)(f(u(r))+g(u(r))) d r\right) d s \\
\int_{\frac{1}{2}}^{1} \varphi_{p}^{-1}\left(\int_{\frac{1}{2}}^{s} q(r)(f(u(r))+g(u(r))) d r\right) d s
\end{array}\right\} \\
& \geq \varphi_{p}^{-1}\left(f\left(\frac{3}{16} R_{1}\right)+g\left(\frac{3}{16} R_{1}\right)\right) \min \left\{\begin{array}{c}
\frac{1}{2} \\
\int_{p}^{-1}\left(\int_{s}^{\frac{1}{2}} q(r) d r\right) d s \\
\int_{\frac{1}{2}}^{1} \varphi_{p}^{-1}\left(\int_{\frac{1}{2}}^{s} q(r) d r\right) d s
\end{array}\right\} \\
& \geq \frac{R_{1}}{C_{2}} \cdot C_{2}=R_{1}=\|u\| .
\end{aligned}
$$

Theorem 1.1 guarantees that there exists $u \in K \cap\left(\bar{\Omega}_{2} \backslash \Omega_{1}\right)$ being a solution to problem (1.3). In addition, from Lemma 1.2, we have $u(t) \geq t(1-t)\|u\| \geq$ $r t(1-t)$ for $t \in[0,1]$.

Case (2): Assume that condition (h8) holds. Essentially the same reasoning as in Case (1) guarantees that there exists $u \in K \cap\left(\bar{\Omega}_{1} \backslash \Omega_{2}\right)$ being a solution to problem (1.3). In addition, from Lemma 1.2, we have $u(t) \geq t(1-t)\|u\| \geq R_{2} t(1-t)$ for $t \in[0,1]$

\section{Remark 3.1.}

(i) The statement in Theorem 3.1 can easily be adjusted so that assumption (H6) can be removed.

(ii) It is easy to use conditions (H7) and (H8) with different constants to obtain a multiplicity result for problem (1.2).

(iii) The ideas in Theorem 3.1 extend so that problem (1.1) could be considered.

\section{References}

[1] Agarwal, R. P. and D. O'Regan: Twin solutions to singular Dirichlet problems. J. Math. Anal. Appl. 240 (1999), 433 - 445.

[2] Agarwal, R. P., Meehan, M. and D. O'Regan: Fixed Point Theory and Applications. Cambridge: Cambridge Univ. Press 2001. 
[3] De Coster, C.: Pairs of positive solutions for the one-dimensional p-Laplacian. Nonlin. Anal. 23 (1994), 669 - 681.

[4] del Pino, M. A., Drábek, P. and R. Manásevich: The Fredholm alternative at the first eigenvalue for the one dimensional p-Laplancian. J. Diff. Equs. 151 (1999), $386-419$.

[5] Haishen Lü and Chengkui Zhong: A note on singular nonlinear boundary value problem for the one-dimensional p-Laplacian. Appl. Math. Letters 14 (2001), 189 - 194.

[6] Haishen Lü and D. O'Regan: A general existence theorem for positive solutions to singular second order ordinary and functional differential equations. Nonlin. Funct. Anal. \& Appl. 5 (2000)2, 1 - 13.

[7] Haishen Lü and D. O'Regan: A general existence theorem for singular equation $\left(\varphi_{p}\left(y^{\prime}\right)\right)^{\prime}+f(t, y)=0$. Math. Inequ. \& Appl. (to appear).

[8] Janus, J. and J. Myjak: A generalized Emden-Fowler equation with a negative exponent. Nonlin. Anal. 23 (1994), 953 - 970.

[9] Junyu Wang and Wenjie Gao: A singular boundary value problem for the onedimensional p-Laplacian. J. Math. Anal. Appl. 201 (1996), $851-866$.

[10] Manasevich, R. F. and F. Zanolin: Time-mappings and multiplicity of solutions for the one-dimensional p-Laplacian. Nonlin. Anal. 21 (1993), $269-291$.

[11] O'Regan, D.: Singular second order boundary value problems. Nonlin. Anal. 15 (1990), 1097 - 1109.

[12] O'Regan, D.: Some general existence principles and results for $\left(\phi\left(y^{\prime}\right)\right)^{\prime}=$ $q f\left(t, y, y^{\prime}\right), 0<t<1$. SIAM J. Math. Anal. 24 (1993), $648-668$.

[13] Taliaferro, S.: On the positive solutions of $y^{\prime \prime}+\phi(t) y^{-\lambda}=0$. Nonlin. Anal. 2 (1978), $437-446$.

[14] Taliaferro, S.: A nonlinear singular boundary value problem. Nonlin. Anal. 3 (1979), $897-904$.

[15] Yong Zhang: Positive solution of singular sublinear Emden-Fowler boundary value problems. J. Math. Anal. Appl. 185 (1994), 215 - 222.

[16] Zhongli Wei: Positive solutions of singular sublinear Emden-Fowler boundary value problems. Systems Sci. \& Math. Sci. 11 (1998), $82-88$.

[17] Zuodong Yang and Zongmin Guo: On the structure of positive solutions for quasilinear ordinary differential equations. Appl. Anal. 58 (1995), 31 - 51.

Received 03.02.2003 